\title{
Métodos para estudo das respostas metabólicas de cães e gatos a diferentes alimentos
}

\author{
Aulus Cavalieri Carciofi ${ }^{1}$
}

\begin{abstract}
1 - Departamento de Clínica e Cirurgia Veterinária, Faculdade de Ciências Agrárias e Veterinárias, Universidade Estadual Paulista, campus de Jaboticabal. Via de Acesso Prof. Paulo Donato Castellane, s/n. Jaboticabal - SP. CEP: 14.884-900. Tel.: (16) 3209-2631. Fax: (16) 3203-1226. E-mail: aulus.carciofi@gmail.com
\end{abstract}

RESUMO - Durante o processo de assimilação e uso dos alimentos, diferentes respostas metabólicas podem ser desenvolvidas pelo organismo dos animais. Estas respostas são fruto da integração de mecanismos complexos, que envolvem os sistemas neuro-endócrino e o funcionamento dos órgãos, sendo influenciadas pela dieta, espécie animal, idade, condição fisiológica e composição corporal. Este trabalho enfoca a importância fisiológica e os métodos de estudo das respostas pós-prandiais aos carboidratos, bem como as alterações fisiológicas conseqüentes ao balanço eletrolítico da dieta. A quantidade, estrutura química e processamento industrial do amido determinam boa parte da resposta pós-prandial de glicose e insulina de cães. Em gatos, outros mecanismos parecem ser mais importantes, como a ingestão de aminoácidos. A fibra alimentar também altera a resposta pós-prandial ao alimento, devendo ser consideradas sua quantidade, solubilidade e fermentabilidade no desenvolvimento das dietas. Os métodos de estudo destas respostas incluem avaliação das respostas glicêmica e insulínca pós-prandiais, teste endovenoso de tolerância à glicose e à arginina. O clâmp euglicêmico apresenta-se também como ferramenta de estudo, no entanto revela informações mais relacionadas ao animal do que à dieta. A compreensão do conjunto de alterações metabólicas aos carboidratos é importante no estudo do controle da saciedade, composição corporal e inúmeras doenças degenerativas e endócrinas. A concentração e relação entre os macro-elementos da dieta ( $\mathrm{Na}, \mathrm{Cl}, \mathrm{K}, \mathrm{P}, \mathrm{Ca}, \mathrm{Mg}$ e S) e dos aminoácidos sulfurados (metionina, cistina e taurina) interferem em inúmeras funções orgânicas, como a cardiovascular, neuromuscular, metabolismo ósseo, renal e pulmonar, refletindo-se no equilíbrio hidro-eletrolítico e ácido-básico orgânicos. De importância prática para cães e gatos encontram-se a relação destes nutrientes com cardiopatias, nefropatias, osteodistrofias e urolitíases. A relação entre os macro-elementos é estabelecida em mmol/kg de matéria seca da dieta, calculando-se seu balanço cátion-ânion (excesso de bases ou ânions dietéticos não determinados). Suas repostas orgânicas são medidas, dentre outros métodos, pela hemogasimetria, balanço hídrico, mensuração do volume dos espaços extracelular e vascular, supersaturação e pH urinários.

Palavras chave: carboidrato, glicose, insulina, metabolismo, $\mathrm{pH}$, urina

\section{Methods of study of metabolic responses of dogs and cats to different foods}

\footnotetext{
ABSTRACT - During the assimilation and use of food process, the animals' organism can develop different metabolic responses. These responses results of complex integration mechanisms, involving the neuro-endocrine systems and organs activity, were being influenced, besides the diet, for the animal species, age, physiologic condition and corporal composition. In this paper will be discussed the physiologic importance and study methods of carbohydrates post-prandial responses, as well as the physiologic alterations induced by diet electrolytic balance. Amount, chemical composition and industrial processing of starch determine treat part of post-prandial glucose and insulin responses in dogs. In cats other mechanisms seem to be more important, including the ingestion of aminoacids. Besides of starch, the dietary fiber also alters the post-prandial response to food. For fiber, it should be considered its amount, solubility and fermentability in development of the diets. Study methods of these responses include glycemic and insulinic meal response test, and intra-venous glucose and arginine tolerance teste. Euglycemic clamp comes, also, as a study tool, however this information says more about the animal than the diet. The understanding of carbohydrates metabolic alterations is important in the study of satiation control, corporal composition and in several degenerative and endocrine diseases. The concentration and relationship among the macro-elements of diet ( $\mathrm{Na}, \mathrm{Cl}, \mathrm{K}, \mathrm{P}, \mathrm{Ca}, \mathrm{Mg}$ and $\mathrm{S})$ and of
} 
sulfurated aminoacids (metionine, cistine and taurine) interfere in many organic functions, as cardiovascular, neuromuscular, bone, renal and lung metabolism, being reflected in organic hydro-electrolyte and acid-base balance. Of practical importance for dogs and cats are the relationship of these nutrients with heart diseases, kidney diseases, osteodystrophy and urolithiasis. The relationship among these macro-elements is established in mmol/kg of diet dry matter, being calculated its cation-anion balance (undetermined anion value). The organic responses is measured, among other methods, by the blood gas analysis, water balance, mensuration of extracelular and vascular spaces volumes, urine supersaturation and urinary $\mathrm{pH}$

Key Words: carbohydrate, glucose, insulin, metabolism, pH, urine

\section{Introdução}

Vários aspectos são importantes na avaliação de um alimento para cães e gatos. Maior ênfase tem sido dada a seu teor de energia metabolizável, digestibilidade, qualidade das fezes geradas mediante seu consumo e palatabilidade. No Brasil, os efeitos metabólicos da dieta e seus possíveis relacionamentos com a saúde geral, no entanto, têm recebido menor atenção.

Os efeitos metabólicos do alimento estão relacionados com alterações de saúde a longo prazo, que podem se estabelecer ao longo de vários meses ou anos de ingestão alimentar. Alguns exemplos incluem as urolitíases, nefropatias, alterações articulares, distúrbios cardio-circulatórios, obesidade, intolerância aos carboidrados (Diabetes Mellitus), dentre outras, todas relacionadas com a qualidade de vida e longevidade de cães e gatos. As respostas metabólicas que mais têm merecido atenção dos pesquisadores são as conseqüentes à ingestão de carboidratos e ao excesso de bases do alimento. Desta forma, este texto traz uma breve revisão sobre a importância e métodos de avaliação das mesmas.

\section{Excesso de bases do alimento e pH urinário}

O balanço cátion-ânion da dieta é importante em vários aspectos do metabolismo, refletindo-se sobre o funcionamento neuromuscular, osteoarticular, função respiratória, função renal e cardiovascular (DiBartola, 1992; Kaneko et al. 1997). Situações de doença, em algumas circunstâncias, levam inclusive a alterações nas necessidades de macro-elementos dietéticos, para que a dieta se adapte melhor às alterações metabólicas por que passa o animal (Hand et al., 2000). Neste texto pretende-se abordar especificamente a relação entre cátions e ânions dietéticos e o pH da urina.
A urina é uma solução complexa e um meio eficiente para a eliminação de produtos de excreção do organismo. É a principal rota pela qual se eliminam produtos do metabolismo protéico (uréia, amônia e creatinina), minerais e água. $\mathrm{O}$ pH urinário varia como consequêencia da manutenção homeostática do equilíbrio ácido-básico (DiBartola, 1992). Em função disso, as características da dieta irão determinar em grande parte $\mathrm{o}$ pH urinário de cães e gatos. A determinação e modulação dietética do $\mathrm{pH}$ urinário, por sua vez, tornam-se importantes devido a seu estreito relacionamento com as urolitíase (Davies, 1999; Osborne et al. 2000, Yamka et al., 2006).

As urolitíases são a causa mais comum de doença obstrutiva do trato urinário inferior em cães e gatos (Wagner et al., 2006), sendo, a formação de cálculos no trato urinário, um problema clínico significativo para animais de companhia em muitos países (Yamka et al., 2006).

No Brasil não existem muitos dados publicados sobre a incidência de desordens urinárias em felinos (Reche Jr et al., 1998). Os 774 cães e gatos atendidos no período de 1999 a 2003 pelo Serviço de Nefrologia e Urologia do Hospital Veterinário "Governador Laudo Natel" da FCAV- Unesp de Jaboticabal, 107 casos foram de urolitíase $(13,82 \%)$ (Camargo, 2004), demonstrando elevada morbidade proporcional.

Em estudo realizado com felinos no Canadian Veterinary Urolith Center, mais de 22.000 casos foram analisados quantitativamente durante 5 anos. Destes, 4866 diagnósticos foram de urolitíase vesical em gatos e 618 plugs uretrais. Aproximadamente 50\% dos urólitos submetidos eram de oxalato de cálcio e $44 \%$ de estruvita (Houston et al., 2003). A formação de urólitos de estruvita é mais comum em gatos jovens e adultos ( 1 a 7 anos), já gatos velhos ( 7 a 9 anos) possuem maior tendência para formar cálculos de oxalato de cálcio (Allen \& Kruger, 2000). Em função disso, as 
características das dietas variam para estas duas faixas etárias.

Esta incidência proporcional de urólitos de estruvita e oxalato de cálcio, no entanto, não pode ser extrapolada para o Brasil. Temos no país uma maior porcentagem de cães e gatos consumindo dietas caseiras, além disso, a composição química dos alimentos industrializados para cães produzidos no Brasil inclui menos proteína e mais cálcio, fósforo e magnésio do que o verificado na Europa e Estados Unidos, como pode ser verificado em Carciofi et al. (2006). Esta composição nutricional, ao menos dos alimentos para cães, sugere que boa parte das rações brasileiras possivelmente leve os animais a produzirem urina alcalina, favorecendo a urolitíase por estruvita. Esta hipótese é reforçada pelos achados de Camargo (2004), em estudo da casuística de urólitos verificada no Hospital Veterinário da FCAV/UNESP. De um total de 105 casos, $24,14 \%$ dos urólitos de cães eram mistos de estruvita e oxalato de cálcio, 24,14\% mistos de estruvita, oxalato de cálcio e hidroxiapatita, 14,94\% urólito puro de oxalato de cálcio e 12,64\% urólitos de estruvita. Em gatos, 61,1\% dos urólitos encontrados foram de estruvita, sendo o restante formações mistas de estruvita com oxalato de cálcio, urato de amônio e cálcio apatita.

\section{Formação dos urólitos}

O risco de desenvolvimento de urolitíase está relacionado a fatores dietéticos e não dietéticos (Zentek et al., 2004). A dieta pode contribuir no aparecimento, manejo ou prevenção de recidivas de urolitíases. Ingredientes da dieta, digestibilidade, composição química e métodos de alimentação afetam o volume, $\mathrm{pH}$ e a gravidade específica da urina (Markwell et al., 1998, Carciofi et al., 2005). Altas concentrações de soluto, com uma subseqüente super saturação da urina e diminuição da freqüência de micção podem favorecer a formação de cristais e cálculos, pois a precipitação de cristais ocorre quando a urina torna-se super saturada (Osborne et al., 2000).

Estratégias dietéticas e de manejo para prevenção e dissolução de urólitos são baseadas nos princípios de saturação da urina. Estas são direcionadas para criar um estado de sub saturação de minerais calculogênicos. A urina contém uma variedade de substâncias que podem inibir ou promover a formação e o crescimento de cristais.
O estado de saturação da urina é o produto da concentração de solutos presentes, $\mathrm{pH}$, força iônica, temperatura e presença de complexos químicos pré-formados. A formação de cristais influencia a precipitação química de íons e moléculas que estão dissolvidos na urina, que se torna super saturada com esses elementos (Allen \& Kruger, 2000). Na Figura 1 encontra-se um esquema da saturação urinária.

A subsaturação é alcançada pela redução na quantidade de precursores de urólitos na dieta, diminuição da concentração de minerais mediante aumento do volume urinário e/ou diminuição de sua excreção urinária e, por fim, por modificação do $\mathrm{pH}$ urinário, medidas estas que aumentam a solubilidade dos cristais.

\section{pH urinário}

Em geral, os urólitos de estruvita se associam em um pH urinário alcalino e os urólitos de oxalato de cálcio, em pH urinário ácido (Allen \& Kruger, 2000). A redução do $\mathrm{pH}$ urinário foi demonstrada como uma prática eficaz na diminuição da incidência de formação de cristais de estruvita, sendo mais importante que a redução do magnésio da dieta (Markwell et al., 1998). Redução de pH, no entanto, pode não ser apropriada para o manejo de outros tipos de urólitos. Dietas acidificantes, que induzem $\mathrm{pH}$ urinário a valores inferiores a 6,29 e apresentam muito pouco magnésio, podem aumentar o risco de formação de cristais de oxalato de cálcio (Markwell et al., 1998). É interessante notar que o magnésio tem um papel protetor, diminuindo a formação de urólitos de oxalato de cálcio (Osborne et al., 2000). O objetivo principal da manipulação dietética é, portanto, atingir um equilíbrio para reduzir o risco de formação destes dois tipos de precipitado.

$\mathrm{O}$ pH urinário apresenta variação circadiana devido à influência de vários fatores como composição do alimento, horário da alimentação e volume consumido (Buffington \& Chew, 1996). Sabe-se que a alimentação ad libitum resulta em onda alcalina pós-prandial de menor magnitude quando comparada à alimentação sob a forma de refeições diárias menos freqüientes. Em conseqüência disso, a interpretação de apenas um valor de $\mathrm{pH}$, especialmente quando não se leva em consideração o momento da alimentação e o tipo de alimento consumido fica bastante duvidosa (Allen \& Kruger, 2000). 


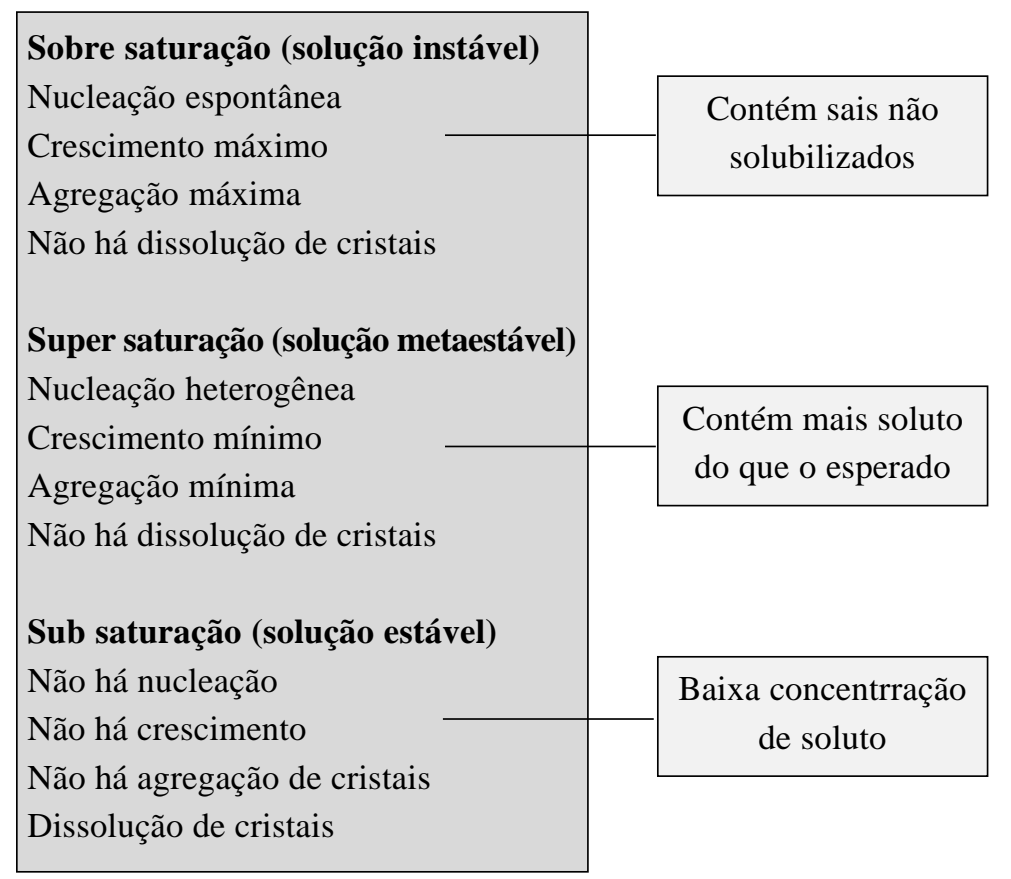

Figura 1 - Eventos prováveis na formação de cristais na urina. Vários fatores influenciam a solubilidade de minerais na urina, incluindo a concentração de minerais litogênicos e não litogênicos, concentração de inibidores e promotores de cristalização, temperatura, $\mathrm{pH}$ e força iônica (adaptado de Osborne et al., 2000).

Figure 1 - Probable events in formation of crystials in urine (Adapted of Osborne et al., 2000).

A ação do alimento sobre o pH urinário é o efeito resultante de seus nutrientes e dos ácidos derivados dos mesmos (Allen \& Kruger, 2000). A maior contribuição de ácidos da dieta é dada pela oxidação de aminoácidos sulfurados e pelo balanço de ânions ( $\mathrm{Cl}, \mathrm{S}, \mathrm{P})$ e cátions $(\mathrm{Na}, \mathrm{Ca}, \mathrm{K}$, Mg) metabolizáveis (Markwell et al., 1998).

Alimentos para cães usados para prevenção de urólitos de estruvita devem levar à produção de urina com pH entre 6,2 e 6,4, enquanto que para a dissolução deste urólito pH entre 5,9 e 6,1. Recomendações para urólitos de oxalato de cálcio são menos precisas em relação ao $\mathrm{pH}$, devendo-se evitar dietas que superacidifiquem a urina (Osborne et al., 2000). Para felinos, recomendam-se alimentos que levem à formação de urina com $\mathrm{pH}$ entre $6,2 \mathrm{e}$ 6,4 para a prevenção de formação e entre pH 5,9 e 6,1 para a dissolução de urólitos de estruvita. Em relação aos urólitos de oxalato de cálcio, estes não podem ser dissolvidos na vesícula urinária, devendo as dietas de prevenção manter pH urinário entre 6,6 e 6,8 (Allen \& Kruger, 2000).

\section{Estimativa do pH urinário a partir da composição da dieta}

๑ 2007 Sociedade Brasileira de Zootecnia
Tendo em vista a grande influência do $\mathrm{pH}$ urinário na prevenção da formação de urólitos, tem surgido interesse no desenvolvimento de métodos de predição do $\mathrm{pH}$ da urina através da composição de macro-elementos e aminoácidos, ou, mais especificamente, composição cátion-iônica do alimento (Kienzle et al., 1991; Zentek et al., 2004). Tais estimativas, se confiáveis, reduzem a necessidade de estudos em animais e permitem otimizar o pH urinário (para prevenção de estruvita e/ou oxalato de cálcio) desencadeado por produtos comerciais. Estas estimativas podem apresentar valor significativo para a indústria de alimentos para animais de companhia, proporcionando diminuição dos custos com testes de alimentos, permitindo ao formulador incluir este parâmetro no desenvolvimento da fórmula da dieta e a comercialização de produtos mais seguros e saudáveis aos animais (Yamka et al., 2006).

A importância e relação da composição mineral da dieta com o metabolismo ácido-básico vem sendo estudada em humanos há mais de 30 anos, sendo apenas mais recentemente considerada para animais (Gevaert et al., 1991). A composição mineral da dieta influencia significativamente o 
fontes de enxofre em alimentos para cães e gatos, como bissulfato de sódio, sulfitos (preservativos), sulfato ferroso, sulfato de manganês, sulfato de condroitina, biotina, tiamina e taurina, que
pH pós-prandial e, portanto, pode predispor cães e gatos a desenvolverem cristalúria ou urolitíase (Kienzle et al., 1991; Zentek et al., 2004). Sais minerais produzem efeito variável sobre o $\mathrm{pH}$ urinário, pois são fontes potenciais de ácido ou base. Os óxidos e carbonatos são alcalinizantes enquanto os cloretos, fosfatos e sulfa-tos produzem efeito acidificante.

Um método prático para se prever o efeito de um alimento sobre o pH urinário é pelo cálculo do excesso de base (EB). Já foi extensamente demonstrado que os cátions e ânions contidos no alimento apresentaram alta correlação com o pH urinário em gatos (Kienzle \& Wilms-Eilers, 1994; Markwell, 1998; Wagner et al., 2006; Yamka et $a l ., 2006)$ e cães (Zentek et al., 1995). Em um estudo com cães, Gevaert et al. (1991) verificaram, além do efeito da composição macromineral de dietas no equilíbrio ácido básico e no pH urinário, a possibilidade de se adicionar acidificantes para a correção de imbalanços minerais, relatando que essa adição pode gerar risco de acidemia crônica. Concluíram que valores de EB devem ser calculados e estabelecidos para a obtenção de um balanço eletrolítico fisiológico em cães, sem o risco de acidemia crônica. Salientaram, ainda, que experimentos in vivo são necessários para avaliar a exata importância de cada macro-mineral no valor do excesso de base.

O EB é calculado a partir das concentrações dos compostos ácidos e alcalinos do alimento, sendo expresso em $\mathrm{mmol} / \mathrm{Kg}$ de matéria seca

\author{
Excesso de bases $(\mathrm{mmol} / \mathrm{kg} \mathrm{MS})=(49,9 \times \mathrm{Ca})+(82,3 \times \mathrm{Mg})+$ \\ $(43,5 \times \mathrm{Na})+(25,6 \times \mathrm{K})-$ \\ $(64,6 \times \mathrm{P})-(86,8 \times \mathrm{S})-\left(28,3 \times \mathrm{Cl}^{*}\right)$ \\ $\mathrm{g} / \mathrm{kg}$ de $\mathrm{MS}$
}

poderiam também interferir no $\mathrm{pH}$ urinário (Yamka et al., 2006). Nesta alternativa, a fórmula seria:

Independentemente da equação empregada, esta essencialmente caracteriza o conteúdo catiônico alcalinizante, como de carbonatos e anions orgânicos, que não foram neutralizados por compostos acidificantes. Quando o enxofre dietético (sulfato) e outros ânions (cloro e fósforo) são absorvidos em maior quantidade, existe uma mudança no equilíbrio cátion-ânion para um estado mais negativo, ou de acidose metabólica. Este estado leva à tendência de aumento da concentração de hidrogênio no sangue arterial. Para que não ocorram diminuições do pH sanguíneo, que permanece sempre levemente alcalino nos animais saudáveis ( $\mathrm{pH} \approx 7,4)$, o organismo lança mão de seus sistemas tampão (Kaneko et al., 1997). Revisão completa sobre estes mecanismos pode ser encontrada em DiBartola (1992). De início atuam os sistemas tampão intracelular, ósseo e respiratório. A longo prazo, no entanto, o equilíbrio é mantido pela maior reabsorção renal de bicarbonato e maior eliminação renal de íons hidrogênio, resultando em queda do $\mathrm{pH}$ da urina.

Carnívoros como cães e gatos são capazes de eliminar grande quantidade de Excesso de bases $(\mathrm{mmol} / \mathrm{kg} \mathrm{MS})=(49,9 \times \mathrm{Ca})+(82,3 \times \mathrm{Mg})+(43,5 \times \mathrm{Na})$ $+(25,6 \times \mathrm{K})-(64,6 \times \mathrm{P})-(13,4 \times$ metionina $)$ - $(16,6 \times$ cistina $)-\left(28,3 \times \mathrm{Cl}^{*}\right)$

* concentração dos elementos em $\mathrm{g} / \mathrm{kg}$ de matéria seca. (Kienzle \& Wilms-Eilers, 1994).

(Allen \& Kruger, 2000). Seu cálculo pode ser realizado, pela fórmula:

Alternativamente, pode-se empregar a concentração de enxofre total do alimento ao invés dos aminoácidos metionina e cistina. Algumas vantagens deste procedimento incluiriam o menor custo da análise de enxofre em relação à de metionina e cistina e a quantificação de outras ácidos. A composição dos alimentos industrializados atuais, no entanto, em muitas ocasiões impõem uma carga catiônica, levando estes animais a produzirem urina neutra ou mesmo alcalina, situação natural para herbívoros, predispondo-os a urolitíase por estruvita (Gevaert, et al., 1991). Kienzle et al., (1991) estudaram o efeito de 10 alimentos comerciais (secos e úmidos) e de alguns aditivos (carbonato de cálcio, lactato de cálcio, fosfato

스 2007 Sociedade Brasileira de Zootecnia 
bicálcico, cloreto de cálcio, ácido fosfórico e cloreto de amônia) sobre o pH urinário de gatos. Os cátions, ânions e aminoácidos que demonstraram associação com o pH urinário foram o cálcio, magnésio, sódio, potássio, metionina, cisteína, fósforo e cloro. Os autores encontraram alta correlação entre os excessos de base e o pH urinário médio dos gatos, podendo ser este estimado como:

\section{pH urina $=6,72+0,0021 \times$ excesso bases $(\mathbf{r}=\mathbf{0 , 9 0 ,} \mathbf{p}<\mathbf{0 , 0 1})$}

Kienzle et al. (1991)

Os autores também sugerem o uso da equação para se determinar o EB desejado para um alimento. Usando-se a equação transformada $[\mathrm{EB}=(\mathrm{pH}$ -6,72)/0,0021 mmol/kg] pode-se prever, durante a formulação, qual EB seria necessário para que o alimento levasse à produção de urina com determinado $\mathrm{pH}$.

Em outro estudo, Kienzle \& Wilms-Eilers (1994) verificaram o efeito do cloreto de amônio e dos carbonatos de cálcio e de sódio no balanço ácido básico em gatos. Os autores confirmaram os resultados do estudo anterior e demonstraram que com a redução gradual do $\mathrm{EB}$, o pH urinário diminui de forma linear até alcançar o valor de 400 a $-500 \mathrm{mmol} / \mathrm{kg}$ de matéria seca, depois do qual não se observaram novas reduções do $\mathrm{pH}$, sendo este descrito por uma regressão quadrática:

\section{pH = 7,1 + 0,0019 $x$ excesso de bases $+(9,7 x$ \\ $\left.10^{-7}\right) \times$ excesso de bases ${ }^{2}(r=0,99, p<0,01)$}

(Kienzle \& Wilms-Eilers, 1994)

Os autores também demonstram redução de $\mathrm{pH}$ sanguíneo em EB muito negativos (elevadas doses de cloreto de amônio) discutindo a importância de se reduzir a apenas o nutricionalmente necessário as concentrações de cátions alcalinizantes, como $\mathrm{Ca}, \mathrm{Mg}, \mathrm{Na}$ e $\mathrm{K}$ do alimento de modo a se trabalhar com uma mínima adição de ânions. Desta forma, reduzir matéria mineral, especificamente cálcio e magnésio, é importante na formulação da dieta. Vômito, apatia e anorexia foram relatados como efeitos secundários em dietas com EB menor que $-1000 \mathrm{mmol} / \mathrm{kg}$. A acidificação orgânica está correlacionada, também, com maior perda renal de fósforo. Deste modo, dietas acidificantes não devem ter relação $\mathrm{Ca}: \mathrm{P}$ maior que $1,1: 1$, sob pena dos felinos desenvolverem hipofosfatemia.

Markwell et al. (1998) estudaram o efeito do balanço entre cátions e ânions sobre o pH urinário de gatos alimentados com 32 diferentes dietas úmidas. Os cátions, ânions e aminoácidos que influenciaram o $\mathrm{pH}$ urinário foram o cálcio, sódio, potássio, fósforo, metionina e cloro. Em estudo mais recente, Wagner et al. (2006) também encontraram correlação positiva entre o excesso de base e o pH urinário médio ao alimentarem gatos com 8 diferentes alimentos industrializados secos. Além disso, os autores correlacionaram o volume urinário de forma positiva com o conteúdo de potássio e de forma negativa com o conteúdo de fósforo, cálcio e magnésio. A equação de predição do $\mathrm{pH}$ urinário encontrada pelos autores $(\mathrm{pH}=6,25+0,0023 \times$ excesso de bases; $\mathrm{r}=0,74$; $\mathrm{p}<0,01)$ foi semelhante à descrita por Kienzle et al. (1991).

Em estudo bastante extenso, Yamka et al. (2006) avaliaram 150 alimentos secos e úmidos para gatos. Encontraram, como nos estudos anteriores, que aumentos de $\mathrm{pH}$ urinário estavam diretamente relacionados com o aumento da ingestão de cálcio, potássio, sódio e magnésio e, a redução do $\mathrm{pH}$ urinário, com maiores teores de enxofre, fósforo e cloro na dieta. Discutem que o enxofre foi o nutriente chave para a diminuição do $\mathrm{pH}$ da urina, tanto para alimentos secos quanto para úmidos e que devem ser usadas fórmulas separadas para alimentos secos e úmidos, com o intuito de se assegurar maior precisão nas estimativas alcançadas. Os autores propuseram uma fórmula direta para a estimativa do $\mathrm{pH}$, sem o calculo prévio do excesso de bases. A fórmula proposta para alimentos secos foi:

$$
\begin{aligned}
& \text { pH da urina }=\mathbf{7 , 0 3}+(\mathbf{1} \times \mathbf{N a})+(\mathbf{1} \times \mathbf{K})+ \\
& (\mathbf{0 , 8 9} \times \mathbf{C a})+(\mathbf{1 , 5 8} \times \mathbf{M g})-(\mathbf{0 , 9 3} \times \mathbf{C l}) \\
& \quad-(\mathbf{1 , 6 1} \times \mathbf{S})-(\mathbf{1 , 0 4} \times \mathbf{P} *) \\
& * \text { concentração dos elementos em g/100g de MS } \\
& \text { Yamka et al. }(2006)
\end{aligned}
$$

O modelo proposto por Yamka et al. (2006) respondeu por $45 \%$ da variabilidade individual de $\mathrm{pH}$ urinários observados e por $70 \%$ da variabilidade média de $\mathrm{pH}$ de uma determinada dieta (média dos gatos que consumiram a dieta). Para o 
conjunto de dados observados (150 rações), as formulas propostas por Kienzle et al. (1991), Kienzle \& Wilms-Eilers (1994) e Markwell et al. (1998) responderam por apenas $25 \%$ a $13 \%$ da variabilidade de $\mathrm{pH}$ urinários, valor considerado baixo. Esta baixa precisão das fórmulas anteriores, segundo os autores, pode ser conseqüente à limitada base de dados empregada nos estudos ou ao fato de outras fontes de enxofre, além da metionina e cistina, não terem sido consideradas nas equações.

$\mathrm{O}$ pH urinários de 12 alimentos secos para gatos produzidos no Brasil foram determinados no Laboratório de Pesquisa em Nutrição e Doenças Nutricionais de Cães e Gatos do DCCV-FCAV/ UNESP. Os resultados obtidos in vivo foram comparados com os previstos por fórmulas publicadas na literatura. Tanto a fórmula preconizada por Yamka et al. (2006) como a de Kienzle \& Wilms-Eilers (1994) apresentaram uma correlação intermediária com os valores in vivo, de respectivamente $r=0,50$ e $r=0,49$, como pode ser visto nas Figuras 2 e 3.

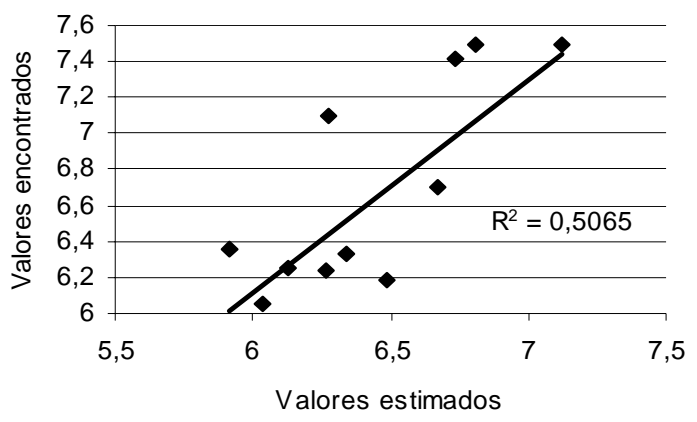

Figura 2 - Correlação entre o pH urinário de gatos encontrado in vivo ( $\mathrm{n}=12$ alimentos) com os estimados pela fórmula de Yamka et al. (2006). Figure 2: Correlation between urinary $\mathrm{pH}$ verified in vivo with urinary $\mathrm{pH}$ estimated according Yamka, et al. (2006).

Para cães, menos estudos foram localizados. As equações de $\mathrm{pH}$ urinário em função do $\mathrm{EB}$, no entanto, não diferem consideravelmente das de felinos. Zentek et al. (1995) sugeriram a seguinte equação para cães:

\section{pH urinário $=\mathbf{6 , 6 1}+\mathbf{0 , 0 0 2} \times$ excesso de bases} Zentek et al. (1995)

\section{Medida in vivo do $\mathrm{pH}$ urinário}

Apesar das equações elencadas servirem de estimativa inicial e serem bastante úteis durante a formulação de um alimento, os resultados in vivo com cães e gatos são, ainda, fundamentais no processo de desenvolvimento e avaliação das rações. Em seu Guia Nutricional Pet a Associação Nacional dos Fabricantes de Alimentos para Animais de Estimação (2007) traz um protocolo mínimo para a determinação do $\mathrm{pH}$ urinário em gatos e recomenda que este seja empregado na avaliação dos produtos. Este mesmo protocolo pode e deve ser utilizado para cães. No anexo 1 apresentamos o protocolo resumido utilizado no Laboratório de Pesquisas em Nutrição e Doenças Nutricionais de Cães e Gatos "Prof. Dr. Flávio Prada" do DCCV-FCAV/UNESP, campus de Jaboticabal. Este visa a coleta de urina sem conservantes. Alem do $\mathrm{pH}$ urinário, de rotina são mensurados o volume diário produzido e a densidade urinária. Protocolos experimentais podem incluir inúmeras outras determinações, a depender das necessidades e objetivos propostos.

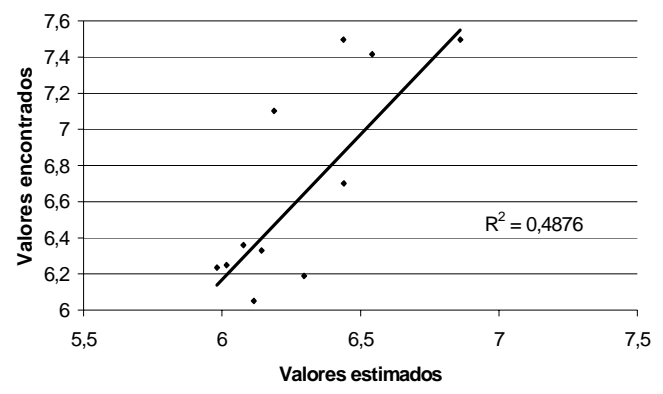

Figura 3 - Correlação entre o $\mathrm{pH}$ urinário de gatos encontrado in vivo ( $\mathrm{n}=12$ alimentos) com os estimados pela fórmula de Kienzle \& WilmsEilers (1994). Figure 3: Correlation between urinary $\mathrm{pH}$ verified in vivo with urinary $\mathrm{pH}$ estimated according Kienzle \& Wilms-Eilers (1994).

Não existe consenso sobre a melhor metodologia para estudo do $\mathrm{pH}$ urinário, o que pode ser verificado pela variação dos protocolos experimentais de diferentes autores. Aspectos importantes a serem considerados são um tempo suficiente de adaptação à dieta, que tem variado de 2 (Kienzle et al., 1991) a 14 dias (Zenteck \& Schultz, 2004). A ocorrência de vômito ou diarréia inviabiliza a avaliação da dieta no animal pois nestes processos a perda de eletrólitos é intensa e alterações no equilíbrio ácido-básico e hidro-eletrolítico se refletem em alterações urinárias importantes.

๑๐ 2007 Sociedade Brasileira de Zootecnia 
O período e a forma de colheita de urina também têm variado entre os experimentos. Períodos de coleta de 14 dias (Wagner et al.,2006), 4 dias (Kienzle et al., 1991) e mesmo 3 dias (Zentek et al., 1995; Yamka et al., 2006) são descritos. Não se localizou estudo que abordasse a variabilidade de $\mathrm{pH}$ urinário entre dias e que determinasse os melhores períodos de adaptação e coleta. Quanto à forma de coleta, a maioria dos estudos realiza coleta total de urina no período de 24 horas, com exceção de Yamka et al. (2006) que trabalharam com o pH médio de duas micções de cada felino no período de 24 horas. Variações circadianas no pH urinário são importantes, principalmente no período pós-prandial, como demonstrado por Kienzle \& Wilms-Eilers (1994). Alimentando os gatos uma única vez ao dia, por 30 minutos, os autores demonstraram no tratamento controle $(\mathrm{EB}=-224 \mathrm{mmol} / \mathrm{kg})$ elevação do $\mathrm{pH}$ urinário $>1 \mathrm{pH} 4$ a 6 horas após a refeição. Demonstraram, também, que estas alterações dependem do EB do alimento, pois a dieta suplementada com carbonato de cálcio $(\mathrm{EB}=$ $+305 \mathrm{mmol} / \mathrm{kg}$ ) apresentou elevação > 1,5 pH, enquanto a suplementada com cloreto de amônia (EB $=-1079 \mathrm{mmol} / \mathrm{kg}$ ) redução do $\mathrm{pH}$ urinário 2 a 4 horas após ingestão alimentar. Em função disso, para muitos tratamentos o $\mathrm{pH}$ da urina produzida no período noturno foi significativamente menor que o da urina produzida durante o dia $(\mathrm{p}<0,05)$.

Por fim, a conservação da urina tem sido feita de diferentes maneiras. Enquanto alguns autores praticam a coleta freqüente, a intervalos de 2 horas, outros a conservam com solução de Thimol (antisséptico) e vaselina ou óleo mineral (evita evaporação), procedimentos que demonstraramse eficazes (Kienzle \& Wilms-Eilers, 1994). Em nossos estudos colhemos a urina diretamente em recipientes plásticos colocados em isopor com gelo, em intervalos regulares, o que reduz a evaporação e o crescimento bacteriano.

\section{Ingestão de carboidratos e respostas glicêmica e insulínica}

O estudo das respostas glicêmicas e insulíncas aos alimentos se remete, em última instância, ao estudo do metabolismo de carboidratos. Características intrínsecas aos próprios carboidratos, como amidos e fibras, bem como dos alimentos, incluindo composição química e processamento, são importantes. Seu estudo é necessário para cães e gatos pois relaciona-se com a manutenção da composição corporal e a incidência de doenças degenerativas. Cães e gatos, animais carnívoros, não têm uma dieta natural rica em amido. Alimentos industrializados secos, entretanto, apresentam de 40 a $55 \%$ de carboidratos na matéria seca (Kronfeld, 1975). No entanto, quando adequadamente processados por extrusão e moagem, cães e gatos apresentam boa capacidade de digerir o amido (de-Oliveira et al., 2006a).

Dentre os fatores do alimento que influenciam a onda pós-prandial de glicose e insulina tem-se: natureza química dos amidos (Goddard et al., 1984; Behall et al., 1989); proteína e gordura da dieta (Nguyen et al., 1994); fibra dietética (Nishimune et al., 1991) e o processamento do alimento (Holste et al., 1989). Em relação à natureza química, o grau de cristalização, a presença de substâncias associadas, como proteínas, e as proporções de amilose e amilo-pectina dos grânulos de amido, afetam sua taxa e velocidade de digestão (Nguyen et al., 1994). Adicionalmente, a quantidade de amido ingerido é, provavelmente, um aspecto também fundamental. Para humanos, Wolever \& Bolognesi (1996) determinaram que a quantidade de amido ingerido corresponde de $46 \%$ a $64 \%$ da variação da glicemia, sendo por vezes inclusive mais importante que o tipo de amido. A quantidade ingerida tem sido imprecisamente descrita em experimentos com cães e gatos, tendo sido pouco explorada sua influência sobre as respostas metabólicas.

A absorção de monossacarídeos resultantes da digestão do amido da dieta constitui uma fonte de glicose sangüínea importante, embora variável. As taxas digestivas e absortivas podem apresentar uma variação alta, mesmo entre animais da mesma espécie e sob regimes dietéticos semelhantes. Após uma refeição rica em carboidratos, as concentrações sangüíneas de glicose podem ficar muito acima daquelas do estado de jejum, mas, num espaço de tempo variável entre espécies, voltam a uma concentração pré-alimentação. Uma segunda fonte contínua de glicose sanguínea, resulta de sua síntese endógena no fígado a partir de aminoácidos gliconeogênicos e glicerol (Beitz, 1996). Alguns estudos sugerem que a gliconeogênese mantêm-se permanentemente ativa em espécies carnívoras estritas como o gato (Case et al., 2000). 
Dados experimentais sugerem que gatos apresentem resposta glicêmica pós-prandial de 18 horas, o que a torna mais prolongada que a de cães e humanos, que apresentam respostas que se completam entre 4 e 6 horas (Bouchard \& Sunvold, 2000; Appleton et al., 2004). As variações das respostas pós-prandiais observadas para gatos indicam que diferenças entre dietas não são tão evidentes. Para essa espécie, ainda não se sabe ao certo qual a participação do amido na determinação das respostas pós-prandiais glicêmicas e insulínicas, parecendo este ter menos contribuição (Kienzle, 1994; Bouchard e Sunvold, 2000; Appleton et al., 2004; de-Oliveira, 2006b).

Esse fato se deve, provavelmente, às diferenças metabólicas dos felinos, dentre elas a utilização prefe-rencial de aminoácidos como fonte de energia e menor habilidade enzimática para digerir e aprovei-tar a glicose dietética, o que torna sua absorção mais lenta e prolongada (Washizu et al., 1999; Kienzle, 1993, 1994; Morris, 2001). Isso também pode ser evidenciado pela observação do tempo de eliminação de glicose após testes orais e intravenosos de tole-rância, sendo este mais prolongado em gatos quando comparados a humanos e cães (Kienzle, 1994; Bouchard e Sunvold, 2000; Appleton et al., 2004).

\section{Alterações do controle glicêmico}

Sabe-se hoje que o controle glicêmico relaciona-se com a expectativa de vida e a incidência de doenças degenerativas. Larson et al. (2003) realizaram um estudo de expectativa de vida em dois grupos de cães. Notaram que os cães que tinham alimentação restrita possuíam menores concentrações de glicose e insulina basal e melhores resultados no teste de tolerância à glicose. Esses achados foram correlacionados positivamente com maior expectativa e qualidade de vida dos cães e com menor incidência de doenças, como a osteoartrite.

Estudos com seres humanos demonstram que a resposta exagerada de insulina plasmática após o consumo de glicose pode estar associada com obesidade, diabetes insulino-independente e doenças cardiovasculares. Alimentos que causam baixa resposta glicêmica pós-prandial foram considerados benéficos em diversos estudos com animais e humanos (Bouchard \& Sunvold, 1998). No entanto, estas informações são bastante escassas para cães e gatos na literatura científica.

O controle glicêmico pode estar prejudicado em alguns estágios fisiológicos e condições de saúde, como diabetes melitus, obesidade, gestação, estresse, infecção, câncer e idade avançada (Nelson et al., 1990; Kahn et al., 2001). A utilização de dietas que minimizem e estendam a onda glicêmica pós-prandial proporcionaria, para animais nestas condições, um restabelecimento mais rápido e fácil da glicemia (Brand-Miller, 1994; Bouchard \& Sunvold, 1999). Protocolos de avaliação das respostas metabólicas pós-prandiais são fundamentais à execução destas pesquisas.

Teoricamente, se propõe que dietas que estimulem uma maior e mais duradoura secreção de insulina contribuam para a excessiva demanda de insulina induzida pela obesidade e que, potencialmente, poderiam contribuir para o desenvolvimento desta doença (Appleton et al., 2004). Além disso, considera-se, em humanos, que dietas que induzem pequenas variações na glicemia e insulinemia aumentam a percepção de saciedade, prevenindo dessa forma a obesidade e podendo ser utilizadas no manejo de pacientes obesos ou diabéticos. Porém, pouco se sabe sobre o uso de carboidratos para animais obesos com problemas no controle glicêmico (Appleton et al., 2004).

Estimativas apontam que a obesidade é a doença nutricional mais comum em cães e gatos, com um prevalência mundial de 35 a $40 \%$ (Scarlett et al.,1994; Bouchard \& Sunvold, 2000). A obesidade, por sua vez, esta fortemente relacionada à intolerância a glicose em gatos (Nelson et al.,1990, Gonçalves, 2006) e cães (Larson et al., 2003). Em dietas para controle de peso recomendase, além do emprego de fontes de amido de digestão lenta, o emprego de concentrações aumentadas de fibra. $\mathrm{O}$ aumento da fibra tem como argumentos: controle da glicemia e lipidemia, redução na digestibilidade dos lipídeos e carboidratos, estimulação dos mecanismos de saciedade, redução na densidade calórica do alimento, manutenção das funções normais do trato gastrintestinal, incluindo qualidade das fezes, tempo de trânsito e absorção da água e eletrólitos no intestino grosso (Burton-Freeman, 2000). Nesta situação deve-se empregar fibras de baixa fermentabilidade, tanto as solúveis como as insolúveis, pois estas podem ser empregadas em

() 2007 Sociedade Brasileira de Zootecnia 
maior quantidade sem ocasionar efeitos digestivos adversos (Butterwick \& Markwell, 1996).

Outros fatores dietéticos que interferem na secreção de insulina e resposta glicêmica

A interpretação das respostas glicêmicas depende de uma avaliação integrada da dieta, incluindo os fatores intrínsecos ao amido, como velocidade de digestão e relação amilose: amilopectina, e os extrínsecos ao amido, como a quantidade ingerida, seu processamento e a composição da dieta (Wolever \& Bolognesi, 1996; Heaton et al., 1988; Brand et al., 1985; Nguyen et al., 1998).

Gulliford et al. (1989) demonstraram que as respostas de glicose sanguínea a alimentos podem ser modificadas pelas ingestões de gordura e proteína. Dietas ricas em gordura retardam o esvaziamento gástrico (Thomas, 1957), reduzindo o aumento da glicose plasmática pós-prandial no homem (Nguyen et al., 1994). Collier et al. (1984) mensuraram o efeito da ingestão da gordura sobre a resposta de glicose a carboidratos de absorção rápida e lenta em indivíduos normais. A ingestão de gordura reduziu a resposta de glicose sanguínea para os dois tipos de carboidratos, embora tenha sido quantitativamente menor para o carboidrato de absorção mais lenta.

Nguyen et al. (1994) demonstraram que os mesmos fatores que contribuem para respostas glicêmicas e insulínicas pós-prandiais em seres humanos influenciam estas respostas nos cães. Conteúdos de proteína e gordura foram determinantes na cinética das respostas glicêmicas e insulínicas nesta espécie. Estes dados, no entanto, não estão disponíveis para a espécie felina.

\section{Métodos de estudo das resposta pós-prandiais de glicose e insulina}

No anexo 2 são descritos resumos dos protocolos para determinação das respostas pósprandias de glicose e insulina empregados no Laboratório de Pesquisas em Nutrição e Doenças Nutricionais de Cães e Gatos "Prof. Dr. Flávio Prada" do DCCV-FCAV/UNESP, campus de Jaboticabal. Protocolos mais complexos estão disponíveis na literatura, podendo ser empregados de acordo com os objetivos experimentais.
Técnica do clamp para determinação da resistência à insulina

Mais recentemente, em Medicina Veterinária, tem-se empregado a técnica de clamp eulgicêmico para o estudo da resistência à insulina. A resistência à insulina (RI) é uma anormalidade metabólica característica de indivíduos com diabetes tipo 2 , diabetes tipo 1 descontrolado, cetoacidose diabética e obesidade. Em populações de não-diabéticos, a redução da ação insulínica pode estar acompanhada de um grupo de alterações metabólicas/cardiovasculares que compreende: hipertensão arterial, hipertrigliceridemia, redução do HDL, colesterol, intolerância aos carboidratos, obesidade centrípeta, aumento de inibidor-1 do ativador do plasminogênio, hiperuricemia e doença cardiovascular aterosclerótica. Este conjunto de alterações da RI é conhecido como síndrome de resistência à insulina ou síndrome metabólica.

O desenvolvimento e aplicação da técnica do clamp de glicose representam seguramente o maior avanço no estudo in vivo da resistência à insulina. Esta técnica permite ao investigador examinar a sensibilidade tecidual à insulina, tanto em músculo como em fígado, bem como a resposta de célula beta à glicose em situações de constância de glicemia e insulinemia. DeFronzo et al. (1979) desenvolveram a técnica do clamp de glicose com suas duas principais variações. A determinação da sensibilidade à insulina pelo clamp é baseada no conceito de que, em condições constantes nas concentrações de glicemia e hiperinsulinemia, a quantidade de glicose consumida pelos tecidos seria igual à quantidade de glicose infundida durante um teste no qual a glicemia é mantida dentro de limites constantes e normais. O teste pressupõe a completa supressão da produção hepática de glicose (Geloneze \& Tambascia, 2006).

A técnica consiste em inserir três cateteres no animal. Em cada veia cefálica é inserido um catéter: um para a infusão contínua de insulina, outro para a infusão de glicose. Na veia jugular é inserido um cateter para colheita de sangue venoso central. A glicemia basal é determinada e então inicia-se a infusão contínua de insulina $(2 \mathrm{mU} / \mathrm{kg} /$ min), com o auxílio de uma bomba de infusão para que a velocidade possa ser controlada. Após 4 minutos do início, inicia-se a infusão contínua de 
glicose a $20 \%$ para que a glicemia basal seja mantida (euglicemia). Para isso, amostras de sangue são colhidas a cada 5 minutos por 1 hora e então a cada 10 minutos para a dosagem de glicemia. A quantidade de glicose injetada é a medida da sensibilidade tecidual à insulina. Para se prevenir hipocalemia, concomitantemente injeta-se fosfato dipotássico na solução de glicose (3mmol/L). O procedimento dura 180 minutos e amostras de sangue para determinação de insulina são colhidos em tubos heparinizados, centrifugados e armazenados $\mathrm{a}-20^{\circ} \mathrm{C}$ até a determinação (Baillanche et al., 2003).

\section{Literatura citada}

ANFAL PET [Associação Nacional dos Fabricantes de Alimentos para Animais de Estimação]. Guia Nutricional de Cães e Gatos. Informativo Técnico. São Paulo, 2007, 72 p.

APPLETON, D.; RAND, J.S.; PRIEST, J. et al. Carbohydrate source affects glucose concentrations, insulin secretion, and food intake in overweight cats. Nutr. Res., v.24, p.447-467, 2004.

BAILLANCHE, E; NGUYEN, P; KREMPF, M. et al. Lipoproteins abnormalities in obese insulin-resistant dogs. Metabolism, v.52, n.5, p.559-564, 2003

BEHALL, K.M.; SCHOFIELD, D.J.; YUHANIAK, I. et al. Diets containing high amylose vs amylopectin starch: Effect on metabolic variables in human subjects. Am. J. Clin. Nutr., v.49, p.337-344, 1989.

BEITZ, D.C. Metabolismo dos carboidratos. In: SWENSON, M.J.; REECE, W.O. (Ed). Dukes fisiologia dos animais domésticos. 11. ed. Rio de Janeiro: Guanabara Koogan, 1996. p.398-411.

BOUCHARD, G.F.; SUNVOLD, G.D. Improving canine glycemic response to a meal with dietary starch. In: THE NORTH AMERICAN VETERINARY CONFERENCE, 1999, Orlando. Proceedings... Orlando, 1999. p. 16-19.

BOUCHARD, G.F.; SUNVOLD, G.D. Effect of dietary carbohydrate source on postprandial plasma glucose and insulin concentration in cats. In: IAMS NUTRITION SYMPOSIUM, 2, 1998, Wilmington. Proceedings... Wilmington, 2000. p. 91-101.

BRAND MILLER, J.C. Importance of glycemic index in diabetes. American Journal of Clinical Nutrition, v.59S, p.747-752, 1994.

BRAND, J.C. et al. Food processing and the glycemic index. American Journal of Clinical Nutrition, v.42, p.1192-1196, 1985.

BUFFINGTON, C.A.T.; CHEW, D.J. Intermittent alkaline urinein a cat fed and acidifying diet. Journal of the American Veterinary Medical Association. p.103-104, 1996.

BUTTERWICK, R.F.; MARKWELL, P.J. Changes in the body composition of cats during weight reduction by controlled dietary energy restriction. The Veterinary Record, V.138, Issue 15, 354-357. 1996.

CAMARGO, C.P. Aspectos clinicos e epidemiológicos de urolitíases em cães e gatos assistidos pelo serviço de nefrologia e urologia da UNESP de Jaboticabal. Dissertação (Mestrado) - Faculdade de Ciências Agrárias e Veterinárias, Universidade Estadual Paulista, Jaboticabal, 55p., 2004.

CARCIOFI, A.C.; VASCONCELLOS, S.; BORGES, N.C. et al. Composição nutricional e avaliação de rótulo de rações secas para cães comercializadas em Jaboticabal-SP. Arquivo Brasileiro de Medicina Veterinária e Zootecnia, v.58, n.3, p.421-426, 2006.

CARCIOFI, A.C.; BAZZOLI, R.S.; ZANNI, A. et al. Influence of water content and the digestibility of pet foods on the water balance of cats. Brazilian Journal Veterinary Research Animal Science. v. 42, n.6, p. 429-434, 2005.

CASE, L.P.; CAREY, D.P.; HIRAKAWA, D.A. Carbohydrates. In: CASE, L.P.; CAREY, D.P.; HIRAKAWA, D.A. Canine and feline nutrition: a resource for companion animal professionals. St. Louis: Mosby-Year Book, 2000. p. 17-94.

COLLIER, G.; MCLEAN, A.; O'DEA, K. Effect of the coingestion of fat on the metabolic responses to slowly and rapidly absorbed carbohydrates. Diabetologia, v.26, p.5054, 1984.

DAVIES, M. Tratamiento de la urolitíases canina y feline. In: BAINBRIDGE, J. \& ELLIOTT, J. Manual de nefrologia y urologia en pequeños animals. Barcelona: Romanya/Valls, S.A., p.275-289, 1999.

DEFRONZO, R.A.; TOBIN, J.D.; ANDRES, R. Glucose clamp technique: a method for quantifying insulin secretion and resistance. Am. J. Physiol. Endocrinol. Metab., v. 237, p. E214-E223, 1979.

DIBARTOLA, S.P.; Fluid therapy in small animal practice. Philadelphia: W.B. Saunders Company, 1992. 719p.

GELONEZE, B.; TAMBASCIA, M.A. Avaliação laboratorial e diagnóstico da resistência insulínica. Arq. Bras. Endocrinol. Metab., v.50, n.2, p. 208-215, 2006.

GEVAERT, D.M.; KLOOSTER, A.T.V.; DE WILDE, R.O. et al. Effect Of Macromineral Composition Of Diets On Blood Acid-Base Equilibrium And Urinary Acidity In Dogs. American Institute Of Nutrition. The Journal of Nutrition, v.121, p.S93-S94, 1991.

GODDARD, M.S.; YOUNG, G.; MARCUS, R. The effect of amylose content on insulin and glucose response to ingested rice. Am. J. Clin. Nutr., v. 42, p.495-503, 1984.

GONÇALVES, K.N.V. Efeito do tratamento da obesidade sobre a glicemia e insulinemia de gatos. Dissertação (Mestrado) - Faculdade de Ciências Agrárias e Veterinárias, Universidade Estadual Paulista, Jaboticabal. 85p, 2006.

GULLIFORD, M.C.; BICKNELL, E.J.; SCARPELLO, J.H. Differential effect of protein and fat ingestion on blood glucose responses to high and low-glycemic index carbohydrates in noninsulin-dependent diabetic subjects. Am. J. Clin. Nutr., v.50, p.773-777, 1989.

HAND, M.S.; THATCHER, C.D.; REMILLARD, R.L. et al. Small Animal Clinical Nutrition. 4.ed. Kansas: Mark Morris Institute, 2000. 1192p.

HEATON, K.W.; MARCUS, S.N.; EMMETT, P.M. et al. Particle size of wheat, maize, and oat test meals: effects on plasma glucose and insulin responses and on the rate of starch digestion in vitro. American Journal of Clinical Nutrition, v.47, p.675-682, 1988.

HOLSTE, L.C.; NELSON, R.W.; FELDMAN, E.C. et al. Effect of dry, soft moist, and canned dog foods on postprandial blood glucose and insulin concentrations in healthy dogs. Am. J. Vet. Res., v.50, p.984-989, 1989.

HOUSTON, D.M.; MOORE, A.E.P.; FAVRIN, M.G. et al. Feline urethral plugs and bladder uroliths: A review of 5484 submissions 1998-2003. Can Vet J., v. 44, 2003.

KAHN, S.E.; PRIGEON, R.L.; ROBERT, S.S. et al. Obesity, body fat distribution, insulin sensitivity and islet b-cell function as explanations for metabolic diversity. Journal of Nutrition., v. 131, p. 354S-360S, 2001.

KANEKO, J.J.; HARVEY, J.W.; BRUSS, M.L. Clinical Biochemistry of Domestic Animals. 5. ed. London: Academic Press, 1997. 932p.

KIENZLE, E. Carbohydrate metabolism of the cat 1. Activity of amylase in the gastrointestinal tract of the cat. J. Anim. Physiol. Anim. Nutr., v. 69, p. 92-101, 1993.

${ }^{\odot} 2007$ Sociedade Brasileira de Zootecnia 
KIENZLE, E. Effect of carbohydrates on digestion in the cat. J. Nutr., v.124, p.2568S-2571S, 1994.

KIENZLE, E.; SCHUKNECHT, A.; MEYER, H. Influence Of Food Composition On The Urine $\mathrm{pH}$ in Cats. American Institute Of Nutrition. The Journal of Nutrition, v.121, p. S87-S88, 1991.

KIENZLE, E; WILMS-EILERS, S. Struvite Diet in Cats: Effect of Ammonium Chloride and Carbonates on Acid Base Balance of Cats. American Institute of Nutrition. The Journal of Nutrition, v.124, p.2652S-2659S, 1994.

KRONFELD, D.S. Nature and use of commercial dog foods. J. Am. Vet. Med. Assoc., v.166, p.487-493. 1975.

KRUGER, J.M.; ALLEN, T.A. Feline lower urinary tract disease. In: HAND, M.S.; TATCHER, C.D.; REMILLARD, R.L.; ROUDEBUSH, P. Small animal clinical nutrition. Missouri: Mark Morris Institute, ed. 4, p.689-724, 2000.

LARSON, B.T.; LAWLER, D.F.; SPITZNAGEL, E.L. et al. Improved Glucose tolerance with lifetime diet restriction favorably affects disease and survival in dogs. Journal of Nutrition, v.133, pp.2887-2892, 2003.

les paràmetres fécaux, la digestibilité des nutriments et les métabolites sanguins. Tesis de doctorado. Univ. de Liège, Bélgica.

MARKWELL, P.J.; BUFFINGTON, C.T.; SMITH, B.H.E. The Effect of Diet on Lower Urinary Tract Diseases in Cats. The Journal of Nutrition, v.128, n.12, pp.2753S-2757S, 1998.

MARKWELL, P.J.; STEVENSON, A.E.; SMITH, B.H.E. A System to Monitor Urinary Tract Health in Dogs. American Society for Nutritional Sciences. The Journal of Nutrition, v.128, p.2761S-2762S, 1998.

MORRIS, J.G. Unique nutrient requirements of cats appear to be diet-induced evolutionary adaptations. Recent Adv. Anim. Nutr. Aust., v.13, p.187-194, 2001.

NELSON, R.W.; HIMSEL, C.A.; FELDMAN, E.C. et al. Glucose tolerance and insulin response in normal weight and obese cats. Am. J. Vet. Res., v.51, p.1357-1362, 1990.

NGUYEN, P.; DUMON, H.; BUTTIN, P. et al. Composition of meal influences changes in postprandial incremental glucose and insulin in healthy dogs. J. Nutr., p.2707S-2711S, 1994.

NISHIMUNE, T.; YAKUSHIJI, T.; SUMIMOTO, T. et al. Glycemic response and fiber content of some foods. Am. J. Clin. Nutr., v.54, p.414-419, 1991.

OLIVEIRA, L.D.; TAKAKURA, F.S.; BAZOLLI, R.S. et al. Dog's and cat's postprandial glycemic and insulinemic responses to different starch souces. In: CONGRESS OF THE EUROPEAN SOCIETY OF VETERINARY AND COMPARATIVE NUTRITION, 10, 2006, Nantes. Congress of the European Society of Veterinary and Comparative Nutrition, 10, 2006. v.2006. p.107-107.
OLIVEIRA, L.D.; TAKAKURA, F.S.; VASCONCELLOS, R.S. et al. Digestibility of starch sources for dogs and cats. In: CONGRESS OF THE EUROPEAN SOCIETY OF VETERINARY AND COMPARATIVE NUTRITION, 10, 2006, Nantes. Congress of the European Society of Veterinary and Comparative Nutrition, 10, 2006. v. 2006. p. 101-101.

OSBORNE, C.A.; BARTGES, J.W.; LULICH, J.P. et al. Prevalence of cystine and urate uroliths in bulldogs and urate uroliths in dalmatians. Journal of the American Veterinary Medical Association, v.204, n.12, p.1914-1918, 2000.

OSBORNE, C.A.; KRUGER, J.M.; LULICH, J.P. et al. Afecções do trato urinário inferior dos felinos.In: ETTINGER, S.J., FELDMAN, E.C. Tratado de Medicina Interna Veterinária - Moléstias do cão e do gato. 4.ed. São Paulo: Manole, p. 1618-1705, 1997.

RECHE Jr., A.; HAGIWARA, M.K.; MAMIZUKA, E. Estudo clínico da doença do trato urinário inferior em gatos domésticos de São Paulo. Braz. J. vet. Res. Anim. Sci., v. 35, n.2, p.69-74, 1998.

SCARLETT, J.M.; DONOQHUE, S.; SAIDLA, J. et al. Overweight cats: Prevalence and risk factors. Int. J. Obes., v.18, suppl. 1, p.22-28, 1994.

THOMAS, J.E. Mechanics and regulation of gastric emptying. Physiol. Rev., v.37, p.453-474, 1957.

WAGNER, E.; KEUSCH, C.; IBEN, C. Influence of the Feed Base Excess on Urine Parameters in Cats. Journal of Animal Physiology and Animal Nutrition, v.90, p.19-24, 2006.

WASHIZU, T.; TANAKA, A.; SAKO, T. et al. Comparison of the activities of enzymes related to glycolysis and gluconeogenesis in the liver of dogs and cats. Res. Vet. Sci., v.67, p.205-206, 1999.

WOLEVER, T.M.S.; BOLOGNESI, C. Prediction of Glucose and Insulin Responses of Normal Subjects after Consuming Mixed Meals Varying in Energy, Protein, Fat, Carbohydrate and Glycemic Index. Journal of Nutrition, v.126, n.11, p.2807-2812. 1996.

YAMKA, R.M.; FRIESEN, K.G.; SCHAKENRAAD, H. The Prediction of urine $\mathrm{pH}$ Using Dietary Cations and Anions in Cats Fed Dry and Wet Foods. Intern J Appl Res Vet Med, v.4, n.1, 2006.

ZENTEK, J; MEYER, H; BEHNSEN, K. Einfluss der Futterzusammentzung auf den Harn-pH beim Hund [Influence of food composition on the urine $\mathrm{pH}$ in the dog]. Kleintierpraxis, v.40, n.1, p. 9-18, 1995.

ZENTEK, J; SCHULZ, A. Urinary Composition of Cats is affected by the Source of Dietary Protein. American Institute Of Nutrition, v.134, p.2162s-2165s, 2004. 


\section{Anexo 1: Protocolo resumido para a determinação do efeito da dieta no pH urinário de cães e gatos}

\section{Preparo dos animais para o teste}

Empregar um mínimo de 6 animais adultos para o teste. Antes do teste os animais deverão ser submetidos ao exame clínico e amostras de sangue e urina deverão ser colhidas para a realização do hemograma e urinálise. Todos os animais empregados no teste deverão estar devidamente desverminados e vacinados. É imprescindível que a avaliação físico-química e do sedimento urinário de cada animal (urinálise) constate, antes do início do teste, perfeita saúde do trato urinário. Dosagens séricas de uréia e creatinina são recomendadas.

A quantidade de alimento fornecida a cada animal pode ser baseada na quantidade necessária para manter o peso corporal ou estimada segundo as necessidades energéticas de manutenção (NRC, 2006). O alimento deverá permanecer disponível durante as 24 horas do dia. Durante o ensaio é importante que a quantidade fornecida de alimento aos animais permaneça constante. Água deverá estar disponível à vontade aos animais durante toda fase experimental.

Os animais deverão permanecer alojados em gaiolas metabólicas individuais em inox ou outro ambiente/material que não interfira com o pH da urina, e que permita a coleta total e quantitativa segura de urina sem contaminação com fezes ou alimento.

\section{Protocolo experimental}

O estudo divide-se em duas fases, adaptação e colheita. O período de adaptação tem duração mínima de sete dias. O período de colheita tem duração mínima de 72 horas. Nesta fase, após sua higienização normal, os ambientes deverão ser lavados ao menos uma vez ao dia e a superfície/ utensílio que terá contato direto com a urina lavado com água destilada e seco com papel toalha. A urina excretada pelos animais deverá ser colhida em recipiente apropriado, identificado, devidamente limpo, enxaguado com água destilada e seco. Estes recipientes deverão permanecer imersos em gelo sob o funil coletor de urina. Outros métodos de conservação da urina podem ser empregados, desde que não haja interferência no $\mathrm{pH}$. A urina deve ser recolhida sempre que eliminada pelos gatos, ou no mínimo quatro vezes ao dia. Este procedimento minimiza a deterioração da amostra e alcalinizações do $\mathrm{pH}$ da mesma resultantes do crescimento de microorganismos.

Imediatamente após seu recolhimento a urina deve ser resfriada entre 4 e $8^{\circ} \mathrm{C}$ em geladeira. A produção urinária individual de cada intervalo de 24 horas deverá ser homogeneizada e ter seu volume quantificado e $\mathrm{pH}$ e densidade determinados. Não deve-se utilizar fitas reagentes para estimativa do $\mathrm{pH}$, deve-se empregar pHmetros digitais de boa procedência. Este procedimento é repetido nos dias seguintes de experimento, tendose ao final no mínimo três valores de volume, $\mathrm{pH}$ e densidade de cada um dos seis animais do ensaio. $\mathrm{O} \mathrm{pH}$ final das $72 \mathrm{~h}$ (mínimo) de colheita de urina será obtido pela média aritmética \pm desvio padrão do valor obtido para cada animal e, conseqüentemente, para o grupo 


\section{Anexo 2: Protocolo resumido para a determinação das respostas pós-prandiais de glicose e insulina}

Cães

A determinação das respostas pós-prandiais de glicose e insulina de cães podem ser realizadas segundo Holste et al. (1989). Recomenda-se adicionar, no entanto, pelo menos mais 2 observações, às 5 e 6 horas após a ingestão do alimento de forma a se abranger efeitos prandiais mais longos. São utilizados, no mínimo, 6 cães por dieta a ser testada, por causa das grandes variações individuais. De preferência utilizam-se cães da mesma raça e idade, sendo fundamental selecionar-se animais com escore corporal entre 4 e 5 (em escala de 1 a 9; Laflamme, 1997a), portanto não obesos.

Um período de 3 dias de adaptação à dieta parece ser suficiente (Nguyen et al., 1994), sendo os animais condicionados a se alimentar em 15 minutos, uma única vez ao dia. O cálculo da quantidade de alimento a ser administrado pode ser feito de acordo com o NRC (2006). Para início do teste, os cães são submetidos a jejum de 24 horas. Na manhã seguinte os cães são cateterizados na veia cefálica cranial, com cateter periférico (Angiocat $^{\circledR}$ ou Jelco ${ }^{\circledast}, 22 \mathrm{GA}$ ), posteriormente fixado com bandagem, sendo os animais mantidos com colar Elizabetano para não retirarem os cateteres.

Após 1 hora de descanso (no mínimo), são colhidas duas amostras de sangue, cuja média será considerada glicemia e insulinemia basais. Após isso, o animal será alimentado com a quantidade de alimento estipulada. O alimento ficará disponível durante 15 minutos. Aquele que não consumir a quantidade total diária de alimento neste período será excluído do teste naquele dia, sendo testado no dia seguinte. Amostras de sangue são colhidas aos 5, 10, 20, 30, 45, 60, 120, 180, $240,300,360$ e 420 minutos após o consumo total do alimento, sendo submetidas às análises das concentrações plasmáticas de glicose e insulina. Como as concentrações de glicose e insulina sofrem variações circadianas, é importante que os horários de coleta de sangue sejam os mesmos para todos os cães.

${ }^{\circledR} 2007$ Sociedade Brasileira de Zootecnia
Gatos

Para felinos pode-se seguir os procedimentos propostos por Appleton et al. (2004). São colhidas duas amostras de sangue em jejum (tempo 0) e após 1, 2, 4, 6, 8, 10, 12, 15 e 18 horas após a ingestão da dieta. São utilizados, no mínimo, 8 gatos por dieta, por causa das grandes variações individuais. De preferência utilizar gatos da mesma raça, idade e com escore corporal entre 4 e 6 (em escala de 1 a 9; Laflamme, 1997b), compatível a um animal normal, não obeso. Como as concentrações de glicose e insulina sofrem variações circadianas, é importante que os horários de coleta de sangue sejam os mesmos para todos os gatos.

$\mathrm{O}$ experimento inicia-se por um período mínimo de 7 dias de adaptação à dieta, quando os gatos são condicionados a se alimentar em 15 minutos, uma única vez ao dia. O cálculo da quantidade de alimentos pode ser conduzido de acordo com NRC (2006). No $5^{\circ}$ dia do período de adaptação, os animais são cateterizados na veia jugular, com cateter central (Intracath, 19 GA (1,1 mm) 8 IN (20,5 cm), Becton Dickinson Vascular Acess, Utah, USA.), mediante anestesia dissociativa. Nos dois dias seguintes os animais são alimentados normalmente, uma vez por dia, sem nenhuma colheita. No terceiro dia após a cateterização (oitavo dia experimental), procedese a determinação das respostas pós-prandiais. São colhidas duas amostras de sangue, cuja média será considerada glicemia e insulinemia basais. Após isso, o animal será alimentado com a quantidade de alimento estipulada. O alimento ficará disponível durante 15 minutos. Aquele que não consumir a quantidade total diária de alimento neste período será excluído do teste naquele dia, sendo testado no dia seguinte.

\section{Manejo das amostras de sangue}

Dos cães se retira cerca de $3 \mathrm{~mL}$ por colheita. Dos gatos, são retirados cerca de $1,5 \mathrm{~mL}$ de sangue por colheita. Essa quantidade é imediatamente dividida em 2 tubos de vidro, um com anticoagulante (Fluoreto), para separação do plasma para mensuração da glicose, e o outro sem anti- 
coagulante, para separação do soro para determinação da insulina. Os tubos são imediatamente centrifugados, sendo o plasma ou soro pipetados e acondicionados em eppendorfs. Para a análise de glicose, os eppendorfs podem ser mantidos em geladeira $\left(2^{\circ} \mathrm{C}\right)$ por 1 dia ou congelados $\left(-15^{\circ} \mathrm{C}\right)$ por 1 semana. Para a análise de insulina, os eppendorfs podem ser congelados $\left(-70^{\circ} \mathrm{C}\right)$ por até 2 meses. A determinação da glicose é, normalmente, realizada pelo sistema enzimático $G O D$ ANA para analisadores automáticos. A Insulina é normalmente dosada por radioimunoensaio, utilizando kits Coat a Count com padrões humanos. É importe verificar junto ao fornecedor do kit se os anticorpos foram testados e validados para felinos, sob pena de se inutilizar os resultados do ensaio. 